\title{
TITLE:
}

\section{Global swing instability of multimachine power systems}

$\operatorname{AUTHOR}(S)$ :

Susuki, Yoshihiko; Mezi ' c, Igor; Hikihara, Takashi

\section{CITATION:}

Susuki, Yoshihiko ... [et al]. Global swing instability of multimachine power systems. Proceedings of the 47th IEEE Conference on Decision and Control 2008: 2487-2492

ISSUE DATE:

2008-12

URL:

http://hdl.handle.net/2433/73438

RIGHT:

(C)2008 IEEE. 


\title{
Global Swing Instability of Multimachine Power Systems
}

\author{
Yoshihiko Susuki, Igor Mezić, Takashi Hikihara
}

\begin{abstract}
This paper studies global instability in swing equations of multimachine power systems. Global instability is related to the undesirable phenomenon of power system, implying that most of all generators in a system coherently lose synchronism with the remaining generators of the system. By analyzing a power system with the loop transmission network, we analytically show that global instability can occur as a result of the interplay between network topology of the system and local dynamics of generators in the system. This suggests a possibility of control for global instability by varying network topology of the system.
\end{abstract}

\section{INTRODUCTION}

Coupled swing dynamics in a population of synchronous machines are of vital importance for power system stability. Transient stability is concerned with the ability of power system to maintain synchronism when subjected to a severe disturbance [1], [2]. Loss of transient stability is recognized as one cause of large blackouts such as the September 2003 blackout in Italy [3], [4]. Transient stability is mainly governed by mechanical characteristics of synchronous machines and is mathematically investigated by nonlinear coupled pendulum-like models, called the classical models or swing equations [1], [2]. Analysis of nonlinear dynamics in the swing equations, e.g. [5], [6], [7], [8], is hence inevitable for prevention of not only the loss of transient stability but also large blackouts.

We study global instability in swing equations of multimachine power systems. Global instability is related to the undesirable phenomenon of power system, implying that most of all generators in a system coherently lose synchronism with the remaining generators of the system. The goal of this study is to delineate a dynamical mechanism to cause blackouts in large-scale power grids. Blackouts spreading into a largescale grid are brought about a sequence of outages [4]. This is partly discussed in [9] with a hybrid dynamical system. A large-scale grid usually consists of strongly innerconnected grids, called subgrids, which are coupled with weak transmission networks. The instability studied here can describe one event in multiple outages, in particular, immediate outage of one subgrid. Then it is possible to model the sequence of subgrid outages as that of global instability. This study is hence expected to explain how the sequence of subgrid outages is dynamically organized and cause large blackouts.

This paper is devoted to analysis of swing equations that represent coupled swing dynamics of a simple power system with the loop topology in Sec. II and the New England test system [10], [11] in Sec. III. Global instability appears in the swing equations for both power systems. In particular, we

This research is supported by JSPS Postdoctoral Fellowships for Research Abroad, 2008-2010.

Y. Susuki is with Department of Electrical Engineering, Kyoto University, Katsura, Nishikyo, Kyoto 615-8510 Japan. He is currently also with Department of Mechanical Engineering, University of California, Santa Barbara. susuki@dove.kuee.kyoto-u.ac.jp

I. Mezic is with Department of Mechanical Engineering, University of California, Santa Barbara, mezic@engineering.ucsb.edu

T. Hikihara is with Department of Electrical Engineering, Photonics and Electronics Science and Engineering Center, Kyoto University, Katsura, Nishikyo, Kyoto 615-8510 Japan. hikihara@kuee.kyoto-u.ac.jp point out for the loop system that incoherent bounded swings of generators lead to their coherent loss of synchronism, namely, global instability. This is motivated by [12], [13], [14] on dynamics of molecular conformation. Sec. II explores a mechanism of occurrence of global instability for the loop system. By mode decomposition and analysis of energy transfer, for a class of disturbances to the loop system, a planar dynamical system is developed that represents collective dynamics for the loop system. This planar system allows one to use phase-plane analysis to investigate global instability. We indeed show that the occurrence of global instability can be predicted by the planar system. The prediction is based on structurally unstable homoclinic orbits that capture both characteristics of the loop topology and generator dynamics. We hence show that global instability can occur as a result of the interplay between network topology of the system and local dynamics of generators in the system. Sec. IV discusses by using the phase-plane analysis a possibility of control for avoiding global instability.

\section{ANALYSis OF Simple LoOP SyStEM}

We analyze a system of swing equations for simple power system in Fig. 1. The power system has the loop topology that is normally adopted in real systems, because the topology makes it easy to deliver electric power. Suppose that $\delta_{i}$ is the rotor angle of generator $i \in\{1, \ldots, N\}$ with respect to the infinite bus, and that $\omega_{i}$ is the rotor speed deviation of generator $i$ relative to the system angular frequency $(2 \pi \times 50 \mathrm{~Hz}$ or $60 \mathrm{~Hz})$, then swing dynamics of generator $i$ are represented by the following system of swing equations:

$$
\left.\begin{array}{rl}
\dot{\delta}_{i}= & \omega_{i} \\
\dot{\omega}_{i}= & p_{\mathrm{m}}-b \sin \delta_{i}+b_{\mathrm{int}}\left\{\sin \left(\delta_{i-1}-\delta_{i}\right)\right. \\
& \left.-\sin \left(\delta_{i}-\delta_{i+1}\right)\right\} .
\end{array}\right\}
$$

$\dot{\delta}_{i}$ denotes the time $(t)$ differentiation of $\delta_{i}$. The boundary conditions for the loop system are $\delta_{0}(t)=\delta_{N}(t)$ and $\delta_{N+1}(t)=\delta_{1}(t)$. The parameters $p_{\mathrm{m}}, b$, and $b_{\text {int }}$ are constant. $p_{\mathrm{m}}$ is the mechanical input power to generator $i, b$

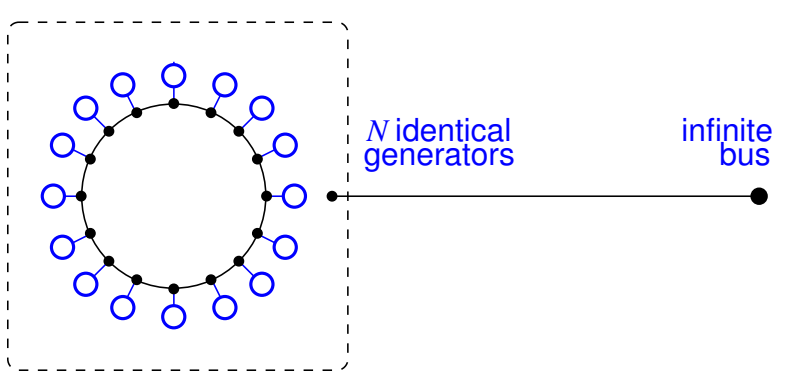

Fig. 1. Simple power system with the loop topology $(N=16)$. The blue circle stands for synchronous machine supplying electric power. The loop system includes $N$ generators, encompassed by the dotted box, and they operate onto the infinite bus [1]. The transmission lines joining infinite bus and generator are much longer than those joining generators in the loop system. It is assumed for modeling that the power system is loss-less 
the critical transmission power between infinite bus and generator $i$, and $b_{\text {int }}$ the critical transmission power between generators $i$ and $i+1$. The system (1) is a mathematical model for the loop system, under the assumption that transformer inductance and synchronous reactance are negligible. The system (1) is also a $N$ degree of freedom Hamiltonian system with well-known Hamiltonian $H\left(\delta_{1}, \ldots, \delta_{N}, \omega_{1}, \ldots, \omega_{N}\right)$ :

$$
H \triangleq \sum_{i=1}^{N} \frac{1}{2} \omega_{i}^{2}-\sum_{i=1}^{N}\left\{\begin{array}{l}
p_{\mathrm{m}} \delta_{i}+b \cos \delta_{i} \\
\left.+b_{\text {int }} \cos \left(\delta_{i-1}-\delta_{i}\right)\right\} .
\end{array}\right.
$$

The contents in Sec. II are given in [15] with their detailed derivation.

\section{A. Global Instability}

Coupled swing dynamics represented by the system (1) are investigated. The parameters for numerical simulations are in per unit system and are given as follows: $p_{\mathrm{m}}=0.009$, $b=0.01, b_{\text {int }}=1$, and $N=20$. The parameter setting has no particular object of real power systems. $p_{\mathrm{m}} / b_{\text {int }}=0.01$ and $b / b_{\text {int }}=0.009$ are much smaller than unity, and we here analyze coupled swing dynamics in the case of strong local transmissions and weak long ones.

Numerical simulations show global instability in the system (1) that is caused by a local disturbance. The local disturbance is given by

$$
\left(\delta_{i}(0), \omega_{i}(0)\right)= \begin{cases}(-1.15,0) & \text { for } i=N / 2 \\ \left(\sin ^{-1} \frac{p_{\mathrm{m}}}{b}, 0\right) & \text { for } i \neq N / 2\end{cases}
$$

where $i=1, \ldots, N$. Physically, generator $N / 2$ is locally disturbed, while the other generators are at their steady states. Fig. 2 shows sequence of rotor angle $\delta_{i}$ for coupled swing dynamics by the local disturbance. At the initial time in Fig. 2(a), generator $N / 2$ is disturbed, and its initial angle $\delta_{N / 2}(0)$ is denoted by the lower red mark. After this, rotor angles of $N$ generators oscillate around their steady states in Figs. 2(b)-(h). These oscillations are incoherent and bounded, although it is not easy to confirm this in Fig. 2. However, they start to show coherent growing in Figs. 2(i)-(p). Finally, they grow unbounded in Figs. 2(q)-(t). The unbounded growing implies that all the generators lose synchronism with the infinite bus. The result hence shows that after a local disturbance, incoherent bounded swings of generators lead to their coherent unbounded growing, that is, global instability.

\section{B. Mechanism}

A mechanism of the occurrence of global instability in Fig. 2 is next investigated. To do so we use two concepts: collective dynamics and a partially linearized system for the system (1).

1) Preliminaries: First, inspired by [12], we define the collective-phase variable $\delta$ and its time derivative $\omega$ as

$$
\delta \triangleq \frac{1}{N} \sum_{i=1}^{N} \delta_{i}, \omega \triangleq \dot{\delta}=\frac{1}{N} \sum_{i=1}^{N} \omega_{i}
$$

Collective dynamics of the loop system are represented by

$$
\dot{\delta}=\omega, \quad \dot{\omega}=p_{\mathrm{m}}-\frac{b}{N} \sum_{i=1}^{N} \sin \delta_{i}
$$

Fig. 3 shows transient behaviors of collective-phase $\delta$ and trajectories of coupled swing dynamics on $\delta-\omega$ plane for local disturbances. The red line for the initial condition
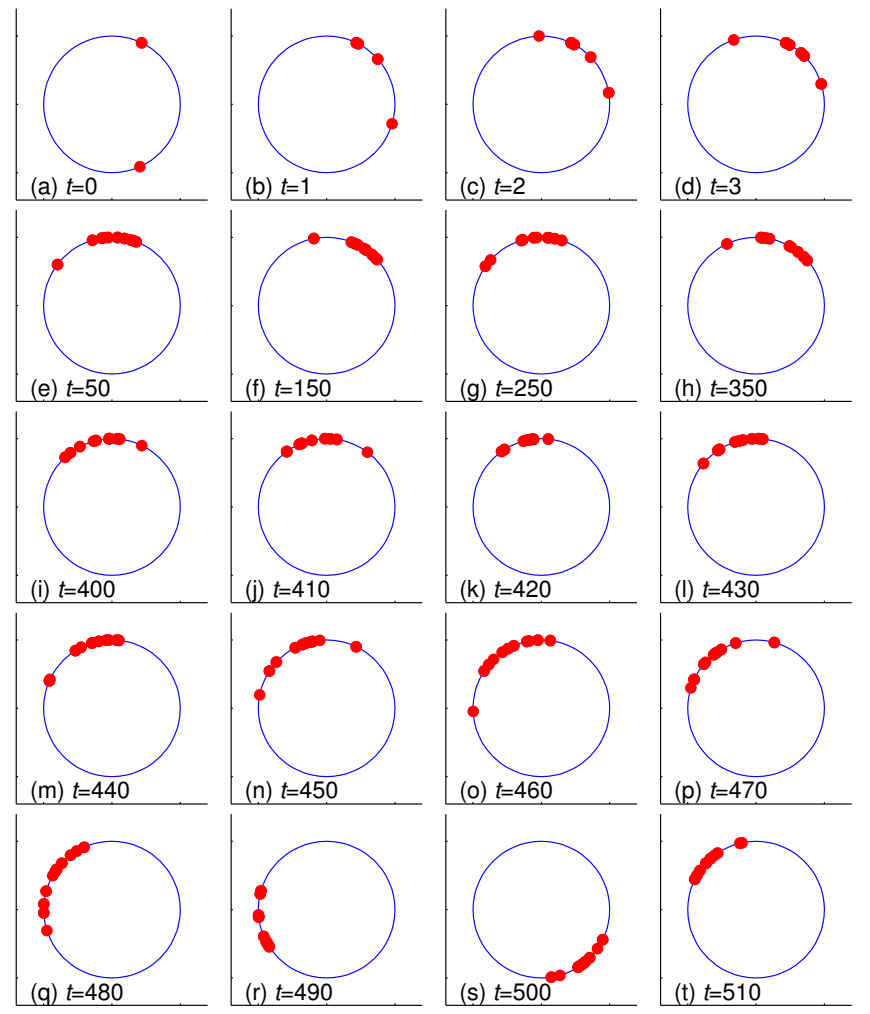

Fig. 2. Sequence of rotor angle $\delta_{i}(t)$ for coupled swing dynamics by the local disturbance. The red marks on blue circle denote the rotor angles of $N$ generators with respect to the infinite bus. The result is obtained with numerical integration of eqs. (1).

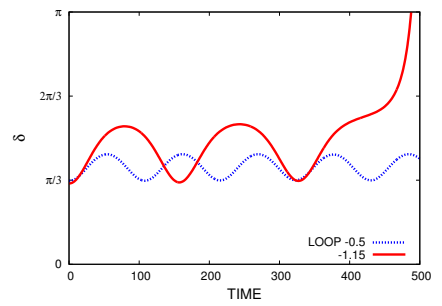

(a) $t-\delta$

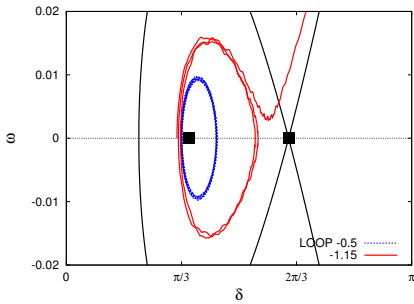

(b) $\delta-\omega$
Fig. 3. Collective dynamics of coupled swings for the local disturbances $\delta_{N / 2}(0)=-0.5$ and -1.15 . The results are obtained for eqs. (1).

$\delta_{N / 2}(0)=-1.15$ corresponds to the instability shown in Fig. 2. The blue line is for $\delta_{N / 2}(0)=-0.5$. The two boxes (ם) denote the equilibrium points of the following system for local dynamics of each generator:

$$
\dot{\delta}=\omega, \quad \dot{\omega}=p_{\mathrm{m}}-b \sin \delta
$$

The system has the saddle point $\left(\pi-\sin ^{-1}\left(p_{\mathrm{m}} / b\right), 0\right)$ connected to itself by a homoclinic orbit $\Gamma_{0}$, denoted by the solid line in Fig. 3(b). For the red trajectory in Fig. 3(b), the intermediate oscillations in Figs. 2(b)-(h) appear as a trapped motion inside $\Gamma_{0}$, and the final coherent growing in Figs. 2(i)-(t) appears as a divergence motion beyond $\Gamma_{0}$. The dynamical picture will play an important role in developing a planar dynamical system for predicting the occurrence of global instability.

Second, we introduce the following system of swing 


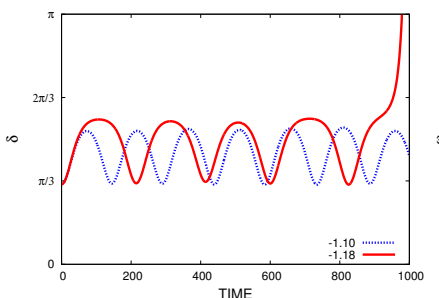

(a) $t-\delta$

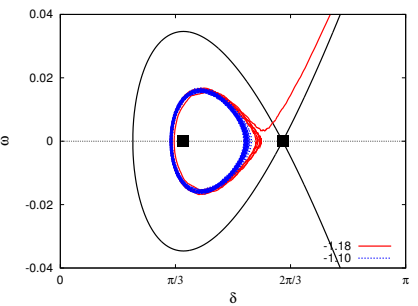

(b) $\delta-\omega$
Fig. 4. Collective dynamics of coupled swings for the local disturbances $\delta_{N / 2}(0)=-1.1$ and -1.18 . The results are obtained for numerical integrations of eqs. (7).

equations with linear coupling:

$$
\left.\begin{array}{rl}
\dot{\delta}_{i}= & \omega_{i}, \\
\dot{\omega}_{i}= & p_{\mathrm{m}}-b \sin \delta_{i}+b_{\mathrm{int}}\left\{\left(\delta_{i-1}-\delta_{i}\right)\right. \\
& \left.-\left(\delta_{i}-\delta_{i+1}\right)\right\} .
\end{array}\right\}
$$

The system corresponds to the discrete sine-Gordon equation system [16], [17], [18] with constant external force $p_{\mathrm{m}}$. This linearized system shows global instability similar to that in the original system (1). Fig. 4 shows transient behaviors of collective-phase $\delta$ and trajectories of coupled swings on $\delta-\omega$ plane for local disturbances. The blue line is for $\delta_{N / 2}(0)=-1.1$, and the red line is for $\delta_{N / 2}(0)=-1.18$. The red lines show behaviors similar to those in Fig. 3 and indeed correspond to global instability in the system (7). We thus explore a mechanism of global instability in the original system (1) through detailed analysis of the system (7) in what follows.

2) Mode Decomposition: Coupled swings represented by the system (7) can be decomposed into a superposition of eigen-oscillations of the loop system. Under $p_{\mathrm{m}}=b=$ 0 , mode of swings in the system (7) is exactly derived. Mode decomposition is based on [17], [16]. Each swing is represented as a superposition of eigen-modes with finite number of eigen-frequencies. The bases $\left\{e_{i j}\right\}_{i, j=1}^{N}$ of eigenmodes and eigen-frequencies $\left\{\Omega_{j}\right\}_{j=1}^{N}$ are given by

$$
\left.\begin{array}{l}
e_{i j} \triangleq \sqrt{\frac{2}{N}} \cos \left(\frac{2 \pi i j}{N}+\frac{\pi}{4}\right), \\
\Omega_{j} \triangleq 2 \sqrt{\left|b_{\text {int }}\right|} \mid \sin \frac{\pi j}{N \mid} .
\end{array}\right\}
$$

Any disturbance, i.e., any set of initial conditions $\left(\delta_{i}(0), \omega_{i}(0)\right)$ for the system (7) with $p_{\mathrm{m}}=b=0$ is represented as a superposition of eigen-modes. By keeping the fact that $p_{\mathrm{m}} / b_{\text {int }}$ and $b / b_{\text {int }}$ are much smaller than unity, it is expected that understanding coupled swings caused by disturbances of eigen-modes leads to clarifying the mechanism.

Figure 5 shows trajectories of coupled swing dynamics for 1 st $(j=1)$ mode disturbance. The mode disturbance is given by

$$
\left(\delta_{i}(0), \omega_{i}(0)\right)=\left(\sin ^{-1} \frac{p_{\mathrm{m}}}{b}+c e_{i, 1}, 0\right) .
$$

$c$ is the strength of mode disturbance. The disturbance implies that at initial time, the loop system is globally perturbed by inner-area 1st mode oscillation. The left box (ם) in Fig. 5 denotes the initial condition of 1 st mode disturbance on $\delta-\omega$ plane. The trajectories with $c=2.5$ and 2.6 show behaviors similar to that in Fig. 4(b), and they are

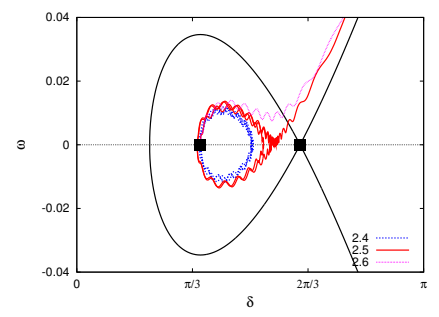

Fig. 5. Collective dynamics of coupled swings for the 1 st mode disturbances with different strengths $c=2.4,2.5,2.6$. The results are obtained for eqs. (7).
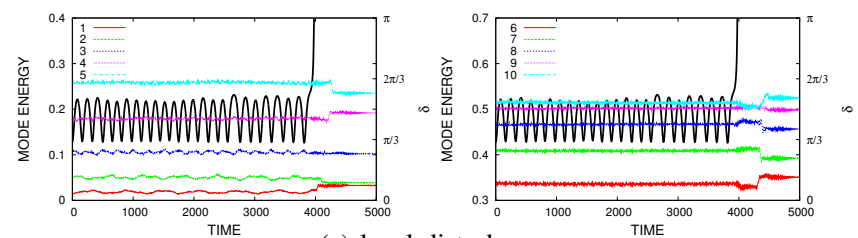

(a) local disturbance

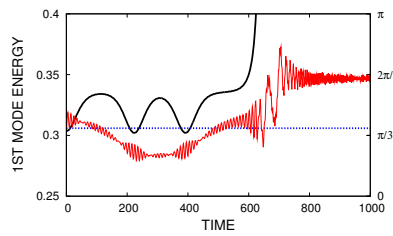

(b) 1st mode disturbance

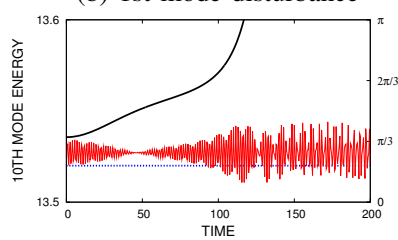

(d) 10th mode disturbance

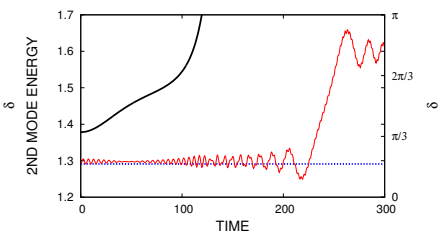

(c) 2nd mode disturbance

Fig. 6. Time evolution of collective- phase $\delta$ (solid line) and mode energy (colored lines). The results are obtained for eqs. (7). There is other mode energy that does not appear in Figs. (b)-(d), because they are of order $10^{-3}$.

indeed global instabilities caused by 1 st mode disturbances in the system (7).

The decomposition using $e_{i j}$ and $\Omega_{j}$ provides important observations of global instability. By mode transformation and action-angle variable one, it is proved that collective dynamics in the system (5) are equivalent to dynamics of non-oscillatory $N$-th $\left(\Omega_{N}=0\right)$ mode. It is also proved that initial energy of modes is localized at the non-oscillatory mode under a coherent solution $\delta_{i}(t)=\delta^{*}(t)(i=1, \ldots, N)$ that well captures the coherent growing in Figs. 2(i)-(t). It is therefore stated that global instability occurs while initial energy is transferred to the non-oscillatory mode.

3) Energy Analysis: The relationship between instability and energy transfer of modes is next investigated. Fig. 6 shows time evolution of collective-phase $\delta$ and mode energy. $n$-th mode energy is defined as the energy contained by $n$-th mode oscillation with angular frequency $\Omega_{n}$ : see [15] for the detailed derivation. The figures are for (a) mode energy in local disturbance at $\delta_{N / 2}(0)=-1.15$, (b) 1 st mode energy in 1st mode disturbance at $c=2.5$, (c) 2nd mode energy in 2nd mode disturbance at $c=2.6$, and (d) 10th mode energy in 10th mode disturbance at $c=2.6$. It should be noted that there is other mode energy that does not appear in Figs. 6(b)(d), because they are of order $10^{-3}$. The figures indicate that there is no dominant energy transfer between oscillatory modes when the collective-phase $\delta$ starts to diverge, namely, global instability appears.

Figure 7 shows time evolution of initial energy in the system (7). The figure is for (a) local disturbance at $\delta_{N / 2}(0)=$ -1.15 , (b) 1 st mode disturbance at $c=2.5$, (c) 2nd mode one at $c=2.6$, and (d) 10th mode one at $c=2.6$. ALL denotes the value of initial energy, namely, the Hamiltonian 


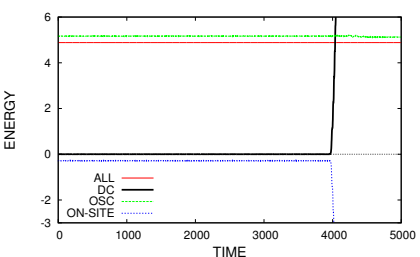

(a) local disturbance

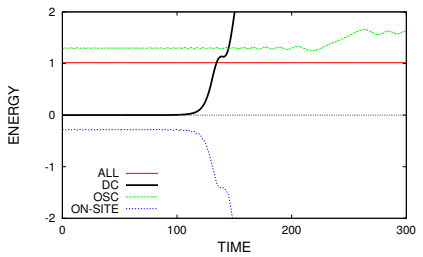

(c) 2nd mode disturbance
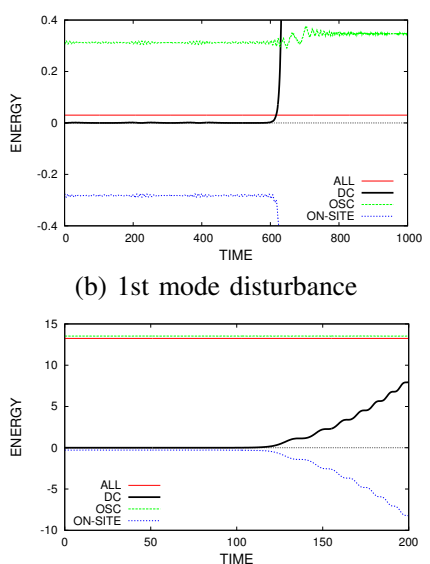

(d) 10th mode disturbance (b) 1st mode disturbance

Fig. 7. Time evolution of energy of the system (7). ALL denotes the value of initial energy, and DC the energy of non-oscillatory (dc) mode. OSC stands for the sum of energy of $N-1$ oscillatory modes, and ON-SITE denotes the sum of energy of on-site potential.

$H$, and DC the energy of non-oscillatory (dc) mode. OSC stands for the sum of energy of $N-1$ oscillatory modes, and ON-SITE denotes the sum of energy of on-site potential $-\sum_{i=1}^{N}\left(p_{\mathrm{m}} \delta_{i}+b \cos \delta_{i}\right)$. The figures indicate that when global instability occurs, the energy of on-site potential is transferred to the non-oscillatory mode. From the above analysis, it is said that the disturbance to single $n$-th oscillatory mode triggers a unidirectional transfer of initial energy to the non-oscillatory mode.

4) Phase-Plane Analysis: For single $n$-th mode disturbance, the above analysis enables us to develop the following planar system that represents global dynamics of nonoscillatory $N$-th mode excited by $n$-th mode oscillation:

$$
\left.\begin{array}{rl}
\dot{\delta} & =\omega, \\
\dot{\omega} & =p_{\mathrm{m}}-\frac{b}{N} \sum_{i=1}^{N} J_{0}\left(e_{i n} c\right) \sin \delta-\frac{b}{N} e(t, \delta) .
\end{array}\right\}
$$

$J_{0}(\cdot)$ denotes the Bessel function, and $e(t, \delta)$ the time- and state-dependent forcing term. Without the forcing term $e$, if $p_{\mathrm{m}}<b \sum_{i=1}^{N} J_{0}\left(e_{i n} c\right) / N$, then the system (10) has one homoclinic orbit $\Gamma_{c}$. Fig. 8 shows $\Gamma_{c}$ for $n=1$, namely, 1st mode disturbance and trajectories of coupled swing dynamics in Fig. 5. If the initial condition (ם) exists inside $\Gamma_{c}$ in Fig. 8(a), then any instability does not occur. If the initial condition (ם) exists outside $\Gamma_{c}$ in Fig. 8(c), then instability occurs. The trajectory near $\Gamma_{c}$ in Fig. 8(b) is qualitatively different for each of Figs. 8(a) and (c).

The instability in Fig. 5 is governed by global dynamics of non-oscillatory mode with homoclinic orbit $\Gamma_{c}$. The intermediate (long) oscillatory swing at $c=2.5$ corresponds to wandering-like motion near the homoclinic orbit $\Gamma_{c}$ of the system (10). $\Gamma_{c}$ here depends on the parameters $p_{\mathrm{m}}$ and $b$ for operating condition of generators and $\left\{e_{i n}\right\}_{i=1}^{N}$ for eigen-mode of the loop system. It is hence said that global instability can occur as a result of the interplay between node dynamics and network topology.

\section{Remarks}

Coherently-synchronized rotational states are presented in [19] for a damped and driven phase model with inertia effect. The phase model is close to the system (1), and the difference is that the phase model has all-to-all coupling structure. The coherent growing in Fig. 2 coincides with the coherent states

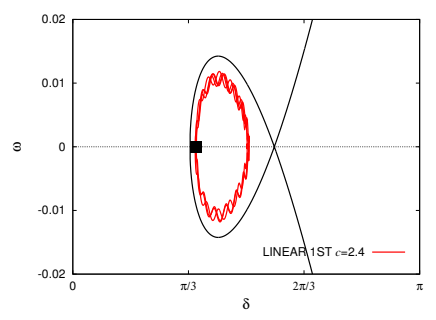

(a) $c=2.4$

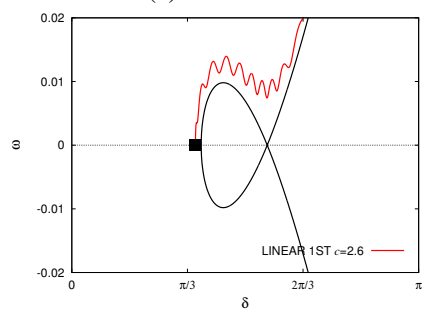

(c) $c=2.6$

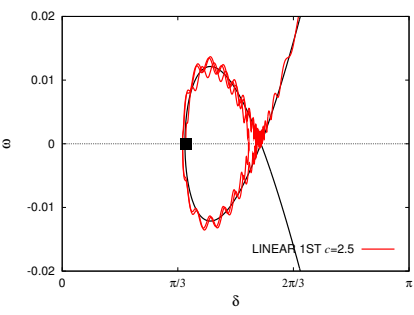

(b) $c=2.5$
Fig. 8. Homoclinic orbit $\Gamma_{c}$ and instability occurrence. The results are obtained for eqs. (7).

of phase model with uniform constant force. It is also shown in [19] that incoherent oscillatory and coherent synchronized rotational states (attractors) coexist in some parameter range. This paper hence addresses the system (1) under a parameter setting of coexisting states and dynamics of transition from incoherent oscillatory state to coherent rotational one.

The above analysis in Sec. II-B is based on the partially linearized system (7). Under some assumptions, the analysis based on mode decomposition is numerically applicable to the original system (1) and shows that the instability in the system (1) can be predicted by the same way as that in the system (7).

The mechanism of instability as unidirectional energy transfer in Sec. II-B would be valid for local disturbances. The prediction above is for mode disturbances, and hence it is not known whether the prediction is possible for the case of local disturbances. Fig. 6 shows that every mode is excited by the local disturbance, and that there is no dominant energy transfer among the $N-1$ modes. By taking all the excited modes into account and tracing the same way of derivation of the system (10), the prediction based on homoclinic orbit would be possible for local disturbances. It is not easy to derive a system similar to the system (10), because multiple mode oscillations affect global dynamics of the non-oscillatory mode.

The above mechanism is not general in eqs. (7), because targeted energy transfer [20], [21] by resonances occurs in the case of weak coupling and can induce conformation change of coupled oscillators [13], [14]. They show phenomena similar to the observed instability in this section and clarify their mechanisms analytically. The difference of mechanisms between [13], [14] and the present result may stem from local dynamics of each oscillator, more precisely, local dynamics under the Morse potential [13], the twinwell potential [14], and the sin-shape potential $V_{\text {local }}\left(\delta_{i}\right) \triangleq$ $-p_{\mathrm{m}} \delta_{i}-b \cos \delta_{i}$.

\section{AnAlysis of the NeW England TeSt System}

We next analyze a system of swing equations in the New England test system in Fig. 9. The test system is widely used for stability studies of power systems. The details of the test system are presented in [11].

\section{A. Swing Equations}

It is assumed that bus 39, onto which machine 1 operates, is the infinite bus. Swing dynamics of generators 2-10 in the 


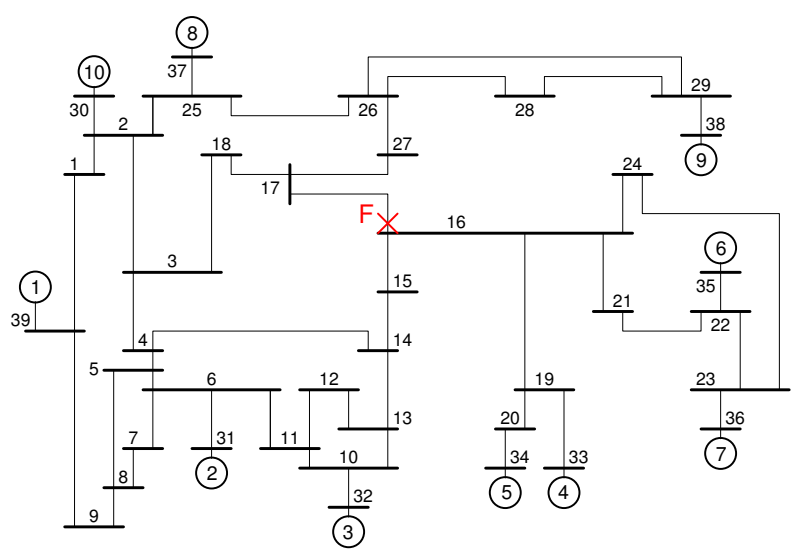

Fig. 9. The New England test system [10], [11]. The system includes 10 synchronous generators and 39 buses. Most of the buses have constant active and reactive power loads. Coupled swing dynamics of 10 generators are studied in the case that a line-to-ground fault occurs at point $F$ near bus 16.

test system can be represented by

$$
\left.\begin{array}{rl}
\dot{\delta}_{i}= & \omega_{i}, \\
\frac{H_{i}}{\pi f_{\mathrm{s}}} \dot{\omega}_{i}= & -D_{i} \omega_{i}+P_{\mathrm{m} i}-G_{i i} E_{i}^{2}-\sum_{\substack{j=1, j \neq i \\
j=1}}^{10} E_{i} E_{j} . \\
& \cdot\left\{G_{i j} \cos \left(\delta_{i}-\delta_{j}\right)+B_{i j} \sin \left(\delta_{i}-\delta_{j}\right)\right\},
\end{array}\right\}
$$

where $i=2, \ldots, 10 . \delta_{i}$ is the rotor angle of generator $i$ with respect to bus 1 , and $\omega_{i}$ the rotor speed deviation of generator $i$ relative to system angular frequency $\left(2 \pi f_{\mathrm{s}}=2 \pi \times 60 \mathrm{~Hz}\right)$. $\delta_{1}$ is constant for the above assumption. The parameters $f_{\mathrm{s}}, H_{i}, P_{\mathrm{m} i}, D_{i}, E_{i}, G_{i i}, G_{i j}$, and $B_{i j}$ are in per unit system except for $H_{i}$ and $D_{i}$ in second, and for $f_{\mathrm{s}}$ in Helz. The mechanical input power $P_{\mathrm{m} i}$ to generator $i$ and the magnitude $E_{i}$ of internal voltage in generator $i$ are assumed to be constant for transient stability studies [1], [2]. $H_{i}$ is the inertia constant of generator $i, D_{i}$ its damping coefficient, and they are constant. $G_{i i}$ is the internal conductance, and $G_{i j}+\mathrm{j} B_{i j}$ the transfer impedance between generators $i$ and $j$; They are the parameters which change with network topology changes. Note that electrical loads in the test system are modeled as passive impedance [11].

\section{B. Numerical Experiment}

Coupled swing dynamics of 10 generators in the test system are simulated. $E_{i}$ and the initial condition $\left(\delta_{i}(0), \omega_{i}(0)=0\right)$ for generator $i$ are fixed through power flow calculation. $H_{i}$ is fixed at the original values in [11]. $P_{\mathrm{m} i}$ and constant power loads are assumed to be $50 \%$ at their ratings [22]. The damping $D_{i}$ is $0.005 \mathrm{~s}$ for all generators. $G_{i i}, G_{i j}$, and $B_{i j}$ are also based on the original line data in [11] and the power flow calculation. It is assumed that the test system is in a steady operating condition at $t=0 \mathrm{~s}$, that a line-to-ground fault occurs at point $F$ near bus 16 at $t=1 \mathrm{~s}-20 /(60 \mathrm{~Hz})$, and that line $16-17$ trips at $t=1 \mathrm{~s}$. The fault duration is 20 cycles of a $60-\mathrm{Hz}$ sine wave. The fault is simulated by adding a small impedance $\left(10^{-7} \mathrm{j}\right)$ between bus 16 and ground. Fig. 10 shows coupled swings of rotor angle $\delta_{i}$ in the test system. The figure indicates that all rotor angles start to grow coherently at about $8 \mathrm{~s}$. The coherent growing is global instability.

\section{Remarks}

It was confirmed that the system (11) in the New England test system shows global instability. A few comments
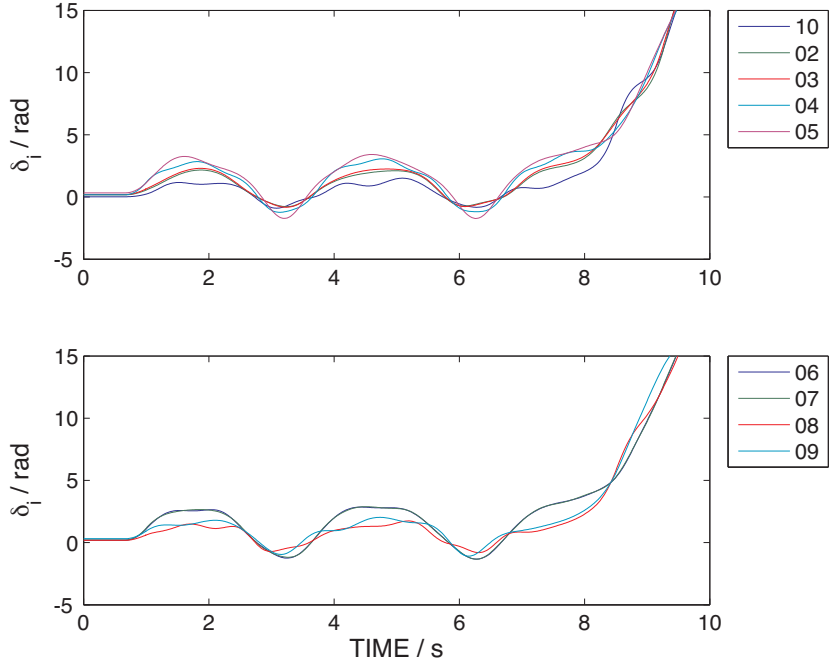

Fig. 10. Coupled swing of phase angle $\delta_{i}$ in New England test system. The fault duration is 20 cycles of a $60-\mathrm{Hz}$ sine wave. The result is obtained by numerical integration of eqs. (11).

are provided to discuss whether the instability in Fig. 10 occurs in the corresponding real power system. First, the classical model with constant voltage behind impedance is used for first swing criterion of transient stability [1]. This is because second and multi swings may be affected by voltage fluctuations, damping effects, controllers such as AVR, PSS, and governor. Second, the fault durations, which we fixed at 20 cycles, are normally less than 10 cycles. Last, the load condition used above is different from the original one in [11]. We cannot hence argue that global instability occurs in the real system. Analysis, however, does show a possibility of global instability in real power systems.

\section{TOWARdS A CONTROL FOR Global Swing INSTABILITY}

Global instability is related to the undesirable phenomenon that should be avoided by control. We introduce a key mechanism for the control problem and discuss control strategies for preventing or avoiding the instability.

\section{A. Internal Resonance as Another Mechanism}

Inspired by [12], we here describe the global instability with dynamical systems theory close to internal resonance [23], [24]. Consider collective dynamics in the system (5). For the system (5) with small parameters $p_{\mathrm{m}}$ and $b$, the set $\left\{(\delta, \omega) \in S^{1} \times \mathbb{R} \mid \omega=0\right\}$ of states in the phase plane is called resonant surface [23], and its neighborhood resonant band. The phase plane is decomposed into the two parts: resonant band and high-energy zone outside of it. Here the initial conditions of local and mode disturbances in Sec. II indeed exist inside the resonant band. The collective motion before the onset of coherent growing is trapped near the resonant band. On the other hand, after the coherent growing, it escapes from the resonant band as shown in Figs. 3(b), 4(b), 5, and 8(b) and (c). The trapped motion is almost integrable and is regarded as a captured state in resonance [23]. At a moment, the integrable motion may be interrupted by small kicks that happen during the resonant band. That is, the so-called release from resonance [23] happens, and the collective motion crosses the homoclinic orbit in Figs. 3(b), 4(b), 5, and 8(b) and (c), and hence it goes away from the resonant band. It is therefore said that global instability 
is release from resonance for collective dynamics of the systems or dynamics of non-oscillatory mode of the systems.

\section{B. Control Strategies}

We discuss how to prevent or avoid the global instability for the loop system. A key mechanism for control appears at the contact between the phase-plane analysis in Sec. II and the above internal resonance. Sec. II showed that the homoclinic orbit $\Gamma_{c}$ in the planar system (10) enables us to predict the instability. Again the above internal resonance suggests that the occurrence is release from resonance for collective dynamics. Therefore we can regard the region encompassed by $\Gamma_{c}$ as a capture region in resonance band for states of collective dynamics. This suggests how to prevent or avoid the occurrence of instability: by some control inputs, we push the collective motion $(\delta(t), \omega(t))$ into the capture region or we enlarge the capture region. A mechanism of the control is by external inputs to avoid the unidirectional energy transfer to the non-oscillatory mode.

There are parameters available as the control inputs of the planar system (10). They are the mechanical input power $p_{\mathrm{m}}$, the critical power $b$ between infinite bus and generator, and the base $\left\{e_{i n}\right\}_{i=1}^{N}$ of $n$-th oscillatory mode. The critical power $b_{\text {int }}$ between generators also becomes a candidate of the control inputs, because it indirectly affects the oscillatory modes. Here, the base $\left\{e_{i n}\right\}_{i=1}^{N}$ and the critical power $b_{\text {int }}$ are effective control inputs. Shedding of generators and lines by protective relays varies the parameters $\left\{e_{i n}\right\}_{i=1}^{N}$ and $b_{\text {int }}$ in the loop system, in other words, the network topology. The facility shedding is one of normal control schemes of power systems. The control of $\left\{e_{i n}\right\}_{i=1}^{N}$, namely, control of oscillatory modes is that it avoids the unidirectional energy transfer to non-oscillatory mode governing coupled swing dynamics.

\section{Conclusions}

This paper analyzed global instability in the swing equations of multimachine power systems. For the loop system, we show that global instability can occur as a result of the interplay between network topology of the system and local dynamics of components in the system. Global instability is related to multi-swing transient instability [2], [25] since the collective motion shows the exit from the separatrix, but the dynamical mechanism is more complicated. The dynamical mechanism gives an analytical explanation of multi-swing instability, which has been recognized as a result of superposition of a slow interarea swing mode and a local-plant swing mode causing a large excursion of rotor angle beyond the first swing [26]. For the loop system, we also point out that linear incoherent bounded swings produce coherent unbounded growing. This scenario for transient instability should be considered in details, because it is hard to identify any critical group of generators in the transient regime, and is hence hard to predict the instability. We next developed a planar dynamical system that can predict the occurrence of instability for the loop system. The prediction is based on structurally unstable homoclinic orbits in the planar system and is similar to traditional methods for single machineinfinite bus system such as energy function methods [11], [2]. Lastly, we discussed a possibility of control of oscillatory modes for the loop system to avoid global instability. The concept of control itself is applicable to various dynamical network models in engineering and science.

\section{ACKNOWLEDGMENTS}

The first author is grateful to Mr. Bryan Eisenhower (University of California, Santa Barbara) for discussions. He is also grateful to Professors Yasuharu Ohsawa and Tsuyoshi Funaki (Kyoto University) for invaluable suggestions of power system analysis and real power systems.

\section{REFERENCES}

[1] E. W. Kimbark, Power System Stability. New York: John Wiley \& Sons, 1947, vol. I.

[2] H. D. Chiang, "Power system stability," in Wiley Encyclopedia of Electrical and Electronics Engineering, J. G. Webster, Ed. New York: John Wiley \& Sons, March 1999, pp. 105-137.

[3] S. Corsi and C. Sabelli, "General blackout in Italy Sunday September 28, 2003, h. 03:28:00," in Proceedings of the IEEE PES General Meeting, vol. 2, Denver, USA, June 2004, pp. 1691-1702.

[4] G. Andersson, P. Donalek, R. Farmer, N. Hatziargyriou, I. Kamwa, P. Kundur, N. Martins, J. Paserba, P. Pourbeik, J. Sanchez-Gasca, R. Schulz, A. Stankovic, C. Taylor, and V. Vittal, "Causes of the 2003 major grid blackouts in North America and Europe, and recommended means to improve system dynamic performance," IEEE Transactions on Power Systems, vol. 20, no. 4, pp. 1922-1928, November 2005.

[5] N. Kopell and R. B. Washburn Jr., "Chaotic motions in the twodegree-of-freedom swing equations," IEEE Transactions on Circuits and Systems, vol. CAS-29, no. 11, pp. 738-746, November 1982.

[6] F. M. A. Salam, J. E. Marsden, and P. P. Varaiya, "Arnold diffusion in the swing equations of a power system," IEEE Transactions on Circuits and Systems, vol. CAS-31, no. 8, pp. 673-688, August 1984.

[7] M. Varghese and J. S. Thorp, "An analysis of truncated fractal growths in the stability boundaries of three-node swing equation," IEEE Transactions on Circuits and Systems, vol. 35, no. 7, pp. 825834, July 1988.

[8] Y. Ueda, T. Enomoto, and H. B. Stewart, "Chaotic transients and fractal structures governing coupled swing dynamics," in Applied Chaos, J. H. Kim and J. Stringer, Eds. London: John Wiley \& Sons, 1992, ch. 8

[9] Y. Susuki, Y. Takatsuji, and T. Hikihara, "Hybrid dynamical system as model for cascading outage in a power system," in Proceedings of the 40th North American Power Symposium, Calgary, Canada, September 28-30 2008.

[10] T. Athay, R. Podmore, and S. Virmani, "A practical method for the direct analysis of transient stability," IEEE Transactions on Power Appratatus and Systems, vol. PAS-98, no. 2, pp. 573-584, March/April 1979.

[11] M. A. Pai, Energy Function Analysis for Power System Stability. Kluwer Academic Pub., 1989.

[12] I. Mezić, "On the dynamics of molecular conformation," Proceedings of the National Academy of Sciences of the USA, vol. 103, no. 20, pp. 7542-7547, 2006.

[13] B. Eisenhower and I. Mezić, "A mechanism for energy transfer leading to conformation change in networked nonlinear systems," in Proceedings of the 46th IEEE Conference on Decision and Control, New Orleans, USA, December 2007, pp. 3976-3981.

[14] - "Actuation requirements of high dimensional oscillator systems," in Proceedings of the 2008 American Control Conference, Seattle, USA, June 2008, pp. 177-182.

[15] Y. Susuki, I. Mezić, and T. Hikihara, "Global swing instability of power grids," (in preparation).

[16] C. G. Goedde, A. J. Lichtenberg, and M. A. Lieberman, "Chaos and the approach to equilibrium in a discrete sine-Gordon equation," Physica D, vol. 59, pp. 200-225, 1992.

[17] M. G. Forest, C. G. Goedde, and A. Sinha, "Instability-driven energy transport in near-integrable, many degrees-of-freedom, Hamiltonian systems," Physical Review Letters, vol. 68, no. 18, pp. 2722-2725, 1992.

[18] S. Watanabe, H. S. J. van der Zant, S. H. Strogatz, and T. P. Orlando, "Dynamics of circular arrays of Josephson junctions and the discrete sine-Gordon equation," Physica D, vol. 97, pp. 429-470, 1996.

[19] H. A. Tanaka, A. J. Lichtenberg, and S. Oishi, "Self-synchronization of coupled oscillators with hysteretic responses," Physica D, vol. 100 pp. 279-300, 1997.

[20] S. Aubry, G. Kopidakis, A. M. Morgante, and G. P. Tsironis, "Analytic conditions for targeted energy transfer between nonlinear oscillators or discrete breathers," Physica B, vol. 296, pp. 222-236, 2001.

[21] P. Maniadis and S. Aubry, "Targeted energy transfer by Fermi resonance," Physica D, vol. 202, pp. 200-217, 2005.

[22] Y. Ohsawa, "Studies on applications of Lypunov's direct method to power system stability analysis," PhD Dissertation, Kyoto University, December 1981, (in Japanese).

[23] A. Neishtadt, "Scattering by resonances," Celestial Mechanics and Dynamical Astronomy, vol. 65, pp. 1-20, 1997.

[24] D. Vainchtein and I. Mezić, "Capture into resonance: A method for efficient control," Physical Review Letters, vol. 93, no. 8, p. 084301, August 2004.

[25] C. C. Chu, "Towards a theory of multi-swing transient instability problems in electric power systems," IEICE Transactions on Fundamentals of Electronics, Communications and Computer Sciences, vol. E88-A, no. 10 , pp. 2692-2695, October 2005.

[26] IEEE/CIGRE Joint Task Force on Stability Terms and Definitions, "Definition and classification of power system stability," IEEE Transactions on Power Systems, vol. 19, no. 2, pp. 1387-1401, May 2004. 